\title{
Repercussões Cardiorrespiratórias da Diálise Peritoneal em Crianças Graves*
}

\author{
Cardiorespiratory Repercussions of the Peritoneal \\ Dialysis in Critically III Children
}

\author{
Patrícia Gombai Barcellos', Cíntia Johnston², Werther Brunow de Carvalho ${ }^{3}$, \\ Marcelo Cunio Fonseca ${ }^{4}$, Jacqueline Evani dos Santos ${ }^{5}$, Eliana Bandini ${ }^{6}$
}

\section{RESUMO}

JUSTIFICATIVA E OBJETIVOS: A diálise peritoneal (DP) é um procedimento freqüentemente indicado para crianças graves com insuficiência renal aguda, sua finalidade é substituir a filtração glomerular e melhorar o controle do equilíbrio hidroeletrolítico e ácido-básico. Entretanto, podem ocorrer alterações cardiorrespiratórias durante o procedimento. Desta forma, o objetivo deste estudo foi descrever as repercussões cardiorrespiratórias da DP em Pediatria

1. Especialista em Fisioterapia Respiratória Pediátrica e Neonatal, UNIFESP-EPM; Supervisora do Curso de Especialização em Fisioterapia Pediátrica e Neonatal e do Curso de Especialização Avançado em Fisioterapia em Emergências e Cuidados Intensivos, UNIFESP-EPM. 2. Doutora em Pediatria e Saúde da Criança, Pontifícia Universidade Católica do Rio Grande do Sul. Chefe do Serviço de Fisioterapia Pediatria, Hospital São Paulo/SPDM; Coordenadora do Curso de Especialização em Fisioterapia Pediátrica e Neonatal e do Curso de Especialização Avançado em Fisioterapia em Emergências e Cuidados Intensivos,UNIFESP-EPM.

3. Professor Adjunto, Livre Docente do Departamento de Pediatria, UNIFESP-EPM; Chefe das Unidades de Cuidados Intensivos Pediátricas do Hospital São Paulo/SPDM, do Hospital SantaCatarina e do Pronto Socorro Infantil Sabará, SP.

4. Médico Intensivista das Unidades de Cuidados Intensivos e SemiIntensivos Pediátricas do Hospital São Paulo/SPDM.

5. Especialista em Fisioterapia Respiratória, EPM-UNIFESP.

6. Especialista em Fisioterapia Respiratória Pediátrica, Instituto da Criança (USP) e em Fisioterapia Cardiorrespiratória, Universidade Metodista de São Paulo; Supervisora do Curso de Especialização em Fisioterapia Pediátrica e Neonatal, UNIFESP/EPM.

*Recebido do Departamento de Pediatria - Unidades de Cuidados Intensivos da Universidade Federal de São Paulo - Escola Paulista de Medicina (UNIFESP-EPM) São Paulo, SP

Apresentado em 22 de maio de 2007

Aceito para publicação em 03 de março de 2008

Endereço para correspondência:

Patrícia Gombai Barcellos

Rua André Rebouças, 87

05109-140 São Paulo, SP

E-mail: pattybarcellos@hotmail.com

(C)Associação de Medicina Intensiva Brasileira, 2008 e o nível de evidência dos estudos que abordam as repercussões.

MÉTODO: Revisão bibliográfica de artigos que abordassem as repercussões cardiorrespiratórias da DP em Pediatria. Pesquisa nos bancos de dados Medline, Ovid e Lilacs, analisando artigos publicados entre 1990 e 2007, com as seguintes palavras-chave, nos idiomas português, inglês e espanhol, respectivamente: diálise peritoneal, efeitos hemodinâmicos, complicações respiratórias, complicações cardíacas, Pediatria; peritoneal dialysis, hemodynamic effects, respiratory complications, cardiac complications, Pediatric; peritoneal diálisis, efecto hemodinámico, complicaciones respiratorias, complicaciones cardiacas, Pediatria. Os artigos encontrados foram classificados em nível de evidências segundo Cook e col. 1992.

RESULTADOS: Foram encontrados 13 artigos, sendo que oito deles abordaram as repercussões cardiocirculatórias e cinco abordaram as repercussões no sistema respiratório da DP. Esses estudos avaliaram pacientes na faixa etária de recém-nascidos a adolescentes graves, totalizando 178 crianças. Dentre as complicações respiratórias mais freqüentes durante a DP encontrou-se a redução da complacência pulmonar e da pressão parcial arterial de oxigênio, aumento da resistência das vias aéreas e da pressão parcial arterial de gás carbônico; após a infusão de fluído na DP os estudos demonstraram um aumento da relação entre a pressão parcial e a fração inspirada de oxigênio, diminuição da diferença alvéolo-arterial de oxigênio e do índice de oxigenação. Dentre as complicações cardiocirculatórias, foram descritas que durante a DP ocorreu um aumento da pressão arterial média, pressão de artéria pulmonar, pressão atrial direita e esquerda, redução da pressão venosa central e aumento da resistência vascular sistêmica. 
CONCLUSÕES: A alteração dos volumes pulmonares, das trocas gasosas e cardiocirculatórias são as repercussões mais freqüentes durante e após a DP em Pediatria. Portanto, as crianças graves com IRA e em DP devem ser monitoradas durante e após este procedimento, para se evitar a piora clínica e para orientação das intervenções multiprofissionais.

Unitermos: Cuidados Intensivos, Diálise Peritoneal, Pediatria.

\section{SUMMARY}

BACKGROUND AND OBJECTIVES: Peritoneal dialysis (PD) is frequently used to replace glomerular filtration and to control acid-base, electrolyte and fluid disturbances in critically ill children with acute renal failure. However, cardiorespiratory changes can happen during this procedure. The objective of this review is to describe the PD cardio-respiratory repercutions in the pediatric patient and the evidence level of the studies that approach these repercutions.

METHODS: Bibliographic revision about PD cardiorespiratory repercutions in the pediatric patient. Medline, Ovid e Lilacs databases were searched for articles from 1990 to 2007 with the following key words in Portuguese, English and Spanish: diálise peritoneal, efeitos hemodinâmicos, complicações respiratórias, complicações cardíacas, Pediatria; peritoneal dialysis, hemodynamic effects, respiratory complications, cardiac complications, Pediatric; peritoneal diálisis, efecto hemodinámico, complicaciones respiratorias, complicaciones cardiacas, Pediatria. The retrieved articles were classified according to Cook et al. 1992. RESULTS: Thirteen articles were retrieved, 8 of them were about cardiorespiratory repercussions and five approached respiratory repercussions of PD. These studies evaluated 178 critically ill patients, from newborns to adolescents. Among the respiratory repercutions during PD the most frequent ones were decrease of the pulmonary compliance and arterial oxygen partial pressure and increase of airway resistance and carbon dioxide partial pressure; after the infusion of PD fluid the studies pointed out an increased arterial oxygen partial pressure/ inspired oxygen fraction relation and diminution of the alveolar-arterial difference and oxygenation index. Increase of the mean arterial pressure, pulmonary artery pressure, right and left atrial pressure and systemic vascular resistance and, reduction of the central venous pressure were the described cardio-circulatory repercutions during PD.

CONCLUSIONS: Pulmonary volumes, gas exchange and cardio-circulatory alterations are the most frequent complications during and after $P D$ in the pediatric patient. Therefore critically ill pediatric patients with acute renal failure needing PD should be monitored during and after this procedure to avoid clinical deterioration and to educate the multi-professional team.

Key Words: Intensive care, Pediatrics, Peritoneal dialysis.

\section{INTRODUÇÃO}

A insuficiência renal caracteriza-se pela diminuição da filtração glomerular com desenvolvimento de uremia progressiva e alterações da homeostase corpórea ${ }^{1}$. Pode ser de origem pré-renal, renal ou pós-renal e o tratamento indicado pode ser a diálise peritoneal (DP), quando principalmente, em insuficiência renal aguda (IRA) não há resposta adequada à terapêutica diurética e/ou inotrópica ${ }^{2}$. IRA é a redução aguda da função renal em horas ou dias. Segundo as Diretrizes de IRA da Sociedade Brasileira de Nefrologia ${ }^{3}$, IRA refere-se principalmente a diminuição do ritmo de filtração glomerular; porém, ocorrem também disfunções no controle do equilíbrio hidroeletrolítico e ácido-básico. Podem ocorrer alterações hormonais, como a deficiência de eritropoetina e de vitamina $D$. O primeiro relato abordando a utilização da DP em Pediatria ocorreu em 1960 por Miller e Finberg ${ }^{4}$. Durante as décadas seguintes, as indicações e o uso desta modalidade aumentaram, sendo atualmente um método freqüentemente utilizado de diálise ${ }^{5}$. A DP, geralmente, é o tratamento de escolha para a insuficiência renal, pois tem baixo custo e necessita de conhecimentos técnicos e operacionais básicos, além de apresentar ótimos resultados para pacientes pediátricos, pois estes têm um aumento da relação entre a superfície peritoneal e o peso corpóreo ${ }^{6}$.

A infusão de líquido na cavidade abdominal ocasiona um aumento pressórico abdominal estimado de $2 \mathrm{cmH}_{2} \mathrm{O}$ a $3 \mathrm{cmH}_{2} \mathrm{O}$ por litro, alterando também a pressão transdiafragmática, podendo repercutir sobre o sistema cardiorrespiratório ${ }^{9}$. A mensuração da pressão intra-abdominal é um parâmetro objetivo para avaliar a tolerância da infusão do dialisato. Em crianças, é recomendado um limite máximo da pressão intra-abdominal de $18 \mathrm{cmH}_{2} \mathrm{O}$, preferencialmente mantendo-a entre $5 \mathrm{cmH}_{2} \mathrm{O}$ e $15 \mathrm{cmH}_{2} \mathrm{O}^{10,11}$. 
Durante e após a infusão de fluidos pela DP podem ocorrer alguns efeitos fisiológicos indesejados (alterações dos volumes pulmonares, trocas gasosas e cardiocirculatórios), sendo importante o reconhecimento destas repercussões para se evitar a descompensação clínica do paciente.

A infusão de fluídos através da DP é um procedimento freqüentemente indicado para pacientes pediátricos graves. Desta forma, o objetivo deste estudo foi descrever as repercussões cardiorrespiratórias mais freqüentes da DP nesta população.

\section{MÉTODO}

Abordou-se as repercussões cardiorrespiratórias da DP em pacientes pediátricos graves. A pesquisa foi realizada nos bancos de dados Medline, Ovid e $\mathrm{Li}$ lacs, selecionando artigos publicados entre 1990 e 2007, com as seguintes palavras-chave, nos idiomas português, inglês e espanhol, respectivamente: diálise peritoneal, efeitos hemodinâmicos, complicações respiratórias, complicações cardíacas, Pediatria; peritoneal dialysis, hemodynamic effects, respiratory complications, cardiac complications, Pediatric; peritoneal diálisis, efecto hemodinámico, complicaciones respiratorias, complicaciones cardiacas, Pediatria. Os artigos encontrados foram classificados por níveis de evidências segundo Cook e col. 1992 (Quadro 1) ${ }^{12}$.

\section{RESULTADOS}

Foram selecionados cinco artigos abordando a DP e as suas repercussões no sistema respiratório, destes, dois artigos avaliaram as repercussões durante a DP, e três após a sua realização. Também foram encontrados 8 artigos abrangendo as repercussões cardiocirculatórias; destes, quatro avaliaram as repercussões durante a DP, quatro após a sua realização, um comparou os efeitos antes e durante a DP. Esses estudos avaliaram desde recém-nascidos a adolescentes com total de amostra de 178 crianças graves. A redução da complacência pulmonar, e da pressão parcial arterial de oxigênio $\left(\mathrm{PaO}_{2}\right)$, o aumento da resistência das vias aéreas, da pressão parcial arterial de gás carbônico $\left(\mathrm{PaCO}_{2}\right)$ foram as repercussões respiratórias mais freqüentes durante a DP; após a DP houve aumento da relação entre a pressão parcial e a fração inspirada de oxigênio $\left(\mathrm{PaO}_{2} / \mathrm{FiO}_{2}\right)$, diminuição da diferença alvéolo-arterial de oxigênio e do índice de oxigenação. Repercussões cardiocirculatórias encontradas durante a DP: aumento da pressão arterial média, da pressão de artéria pulmonar, da pressão atrial direita e esquerda, redução da pressão venosa central e aumento da resistência vascular sistêmica. As tabelas 1 e 2 demonstram os artigos encontrados, a classificação do nível de evidência científica e os seus resultados.

Quadro 1 - Classificação das Evidências Científicas

\begin{tabular}{ll}
\hline \multicolumn{2}{l}{ Classificação das Respostas aos Questionamentos } \\
\hline A & Suportadas por pelo menos duas investigações de nível I \\
B & Suportadas por somente uma investigação de nível I \\
C & Suportadas somente por investigações de nível II \\
D & Suportadas por pelo menos uma investigação de nível III \\
E & Suportadas por evidências de nível IV ou V \\
Nível das Evidências & Estudos aleatórios; grande número de pacientes; resultados claros; risco baixo de erro alfa (falso-positivos) ou beta (falso- \\
Nível I & negativos) \\
Nível II & $\begin{array}{l}\text { Estudos aleatórios; pequeno número de pacientes; resultados incertos; risco moderado a elevado de erro alfa } \\
\text { (falso-positivo) ou beta (falso-negativo) }\end{array}$ \\
Nível III & Estudos não aleatórios, controles contemporâneos \\
Nível IV & Estudos não aleatórios, controles históricos e opinião de especialistas \\
Nível V & Séries de casos; estudos não-controlados e opinião de especialistas
\end{tabular}


Tabela 1 - Estudos sobre as Repercussões Respiratórias da Diálise Peritoneal

\begin{tabular}{|c|c|c|c|}
\hline Autores & $\begin{array}{l}\text { Delineamento } \\
\text { do Estudo }\end{array}$ & Amostra & Resultados \\
\hline $\begin{array}{l}\text { Sagy e } \\
\text { Silver }^{26}\end{array}$ & Nível IV & $\begin{array}{l}6 \text { crianças } \\
\text { Idade (meses): } 18,7 \pm 37 \\
\text { Diagnóstico: IRA em SDRA }\end{array}$ & $\begin{array}{l}\text { Aumento da relação } \mathrm{PaO}_{2} / \mathrm{FiO}_{2} \text {, redução da diferença alvéolo-arterial de } \\
\mathrm{O}_{2} \text { e do índice de oxigenação após a indicação e utilização da DP. }\end{array}$ \\
\hline $\begin{array}{l}\text { Morris } \\
\text { Butt e } \\
\text { Karl }\end{array}$ & Nível IV & $\begin{array}{l}6 \text { crianças } \\
\text { Idade (meses): } 35(2-11,5) \\
\text { Diagnóstico: IRA em PO de cor- } \\
\text { reção de cardiopatia congênita }\end{array}$ & $\begin{array}{l}\text { Quando administrado diversos volumes de dialisato }(0,10,20 \text { e } 30 \mathrm{~mL} / \\
\mathrm{kg}) \text { não houve alterações significativas na função respiratória e na oxi- } \\
\text { genação. }\end{array}$ \\
\hline $\begin{array}{l}\text { Werner } \\
\text { e col. }{ }^{25}\end{array}$ & Nível V & $\begin{array}{l}32 \text { crianças } \\
\text { Idade (meses): } 22 \pm 35 \\
\text { Diagnóstico: IRA em PO de cor- } \\
\text { reção de cardiopatia congênita }\end{array}$ & $\begin{array}{l}\text { Redução da pressão média de vias aéreas e da diferença alvéolo-arterial } \\
\text { de oxigênio após a administração da DP. }\end{array}$ \\
\hline $\begin{array}{l}\text { Bokariia } \\
\text { e col. }{ }^{24}\end{array}$ & Nível V & $\begin{array}{l}19 \text { recém-nascidos e crianças } \\
\text { Idade (meses): } 6,2 \pm 4,3\end{array}$ & $\begin{array}{l}\text { Melhora hemodinâmica, de troca gasosa e função pulmonar após } 4 \text { a } 5 \\
\text { dias de DP. }\end{array}$ \\
\hline $\begin{array}{l}\text { Bunch- } \\
\text { man e } \\
\text { col. }^{19}\end{array}$ & Nível V & $\begin{array}{l}4 \text { crianças } \\
\text { Diagnóstico: IRA em pacientes } \\
\text { submetidos à ventilação pulmo- } \\
\text { nar mecânica. }\end{array}$ & $\begin{array}{l}\text { Diminuição da complacência pulmonar e aumento de resistência das vias } \\
\text { aéreas durante a administração do dialisato e aumento da pressão intra- } \\
\text { abdominal na DP com concomitante diminuição da pressão parcial arte- } \\
\text { rial de oxigênio e aumento da pressão arterial de dióxido de carbono. }\end{array}$ \\
\hline
\end{tabular}

$\mathrm{DC}=$ débito cardíaco; $\mathrm{DP}=$ diálise peritoneal; $\mathrm{IRA}=$ insuficiência renal aguda; $\mathrm{O}_{2}=$ oxigênio; $\mathrm{PAM}=$ pressão arterial média; $\mathrm{PO}=$ pós-operatório; $\mathrm{PVC}=$ pressão venosa central; RVS= resistência vascular sistêmica; SDRA= síndrome do desconforto respiratório agudo.

Tabela 2 - Estudos sobre as Repercussões Cardiocirculatórias da Diálise Peritoneal

\begin{tabular}{|c|c|c|c|}
\hline Autores & $\begin{array}{l}\text { Delineamento } \\
\text { do Estudo }\end{array}$ & Amostra & Resultados \\
\hline $\begin{array}{l}\text { Dittrich e } \\
\text { col. }^{27}\end{array}$ & Nível V & $\begin{array}{l}6 \text { crianças } \\
\text { Idade (meses): } 1,1(0,2-11,8) \\
\text { Diagnóstico: IRA em PO de correção de cardio- } \\
\text { patia congênita }\end{array}$ & $\begin{array}{l}\text { Aumento da PAM, pressão de artéria pulmonar, de } \\
\text { átrio esquerdo e PVC durante a DP, sem efeitos no DC } \\
\text { e na resistência vascular sistêmica. }\end{array}$ \\
\hline $\begin{array}{l}\text { Dittrich e } \\
\text { col. }^{31}\end{array}$ & Nível IV & $\begin{array}{l}27 \text { crianças } \\
\text { Idade (meses): 1,4 }(0,1-11,8) \\
\text { Diagnóstico: IRA em PO de correção de cardio- } \\
\text { patia congênita }\end{array}$ & $\begin{array}{l}\text { Após a DP houve aumento da PAM, redução da PVC e } \\
\text { menor necessidade do uso da epinefrina. }\end{array}$ \\
\hline $\begin{array}{l}\text { Morris, Butt } \\
\text { e Karl'23 }\end{array}$ & Nível IV & $\begin{array}{l}6 \text { crianças } \\
\text { Idade (meses): } 35 \text { (2 -11,5) } \\
\text { Diagnóstico: IRA em PO de correção de cardio- } \\
\text { patia congênita }\end{array}$ & $\begin{array}{l}\text { Quando administrado diversos volumes de dialisato } \\
(10,20 \text { e } 30 \mathrm{~mL} / \mathrm{kg} \text { ) o índice cardíaco foi maior quando } \\
\text { a cavidade abdominal estava vazia e com } 10 \mathrm{~mL} / \mathrm{kg} \text {. }\end{array}$ \\
\hline $\begin{array}{l}\text { Werner e } \\
\text { col. }^{25}\end{array}$ & Nível V & $\begin{array}{l}32 \text { crianças } \\
\text { Idade (meses): } 22 \pm 35 \\
\text { Diagnóstico: IRA em PO de correção de cardio- } \\
\text { patia congênita }\end{array}$ & $\begin{array}{l}\text { Aumento da PAM, diminuição da PVC após a adminis- } \\
\text { tração da DP. }\end{array}$ \\
\hline $\begin{array}{l}\text { Bokariia e } \\
\text { col. } .^{24}\end{array}$ & Nível V & $\begin{array}{l}19 \text { recém-nascidos e crianças } \\
(6,2 \pm 4.3 \text { meses })\end{array}$ & $\begin{array}{l}\text { Melhora hemodinâmica, troca gasosa e função pulmo- } \\
\text { nar após } 4 \text { a } 5 \text { dias de DP. }\end{array}$ \\
\hline $\begin{array}{l}\text { Bunchman } \\
\text { e col. }{ }^{19}\end{array}$ & Nível V & $\begin{array}{l}59 \text { crianças em DP } \\
\text { Idade (meses): } 74 \pm 11,7 \\
\text { Diagnósticos: IRA após transplante de medula } \\
\text { óssea, síndrome da lise tumoral, cardiopatia } \\
\text { congênita, síndrome hemolítico-urêmica, necro- } \\
\text { se tubular aguda e após transplante hepático. }\end{array}$ & $\begin{array}{l}\text { Aumento da pressão de átrio direito e pressão arte- } \\
\text { rial pulmonar durante a DP, sem alteração do índice } \\
\text { cardíaco, pressão arterial média, resistência vascular } \\
\text { pulmonar e sistêmica e saturação arterial de oxigênio. }\end{array}$ \\
\hline $\begin{array}{l}\text { Zhovnir e } \\
\text { col. }^{30}\end{array}$ & Nível V & $\begin{array}{l}16 \text { crianças } \\
\text { Diagnóstico: IRA em PO de correção de cardio- } \\
\text { patia congênita }\end{array}$ & $\begin{array}{l}\text { Melhora nos parâmetros hemodinâmicos, renais e pul- } \\
\text { monares. }\end{array}$ \\
\hline $\begin{array}{l}\text { Ryan, Hung } \\
\text { e Soder } 29\end{array}$ & Nível V & $\begin{array}{l}3 \text { crianças } \\
\text { Diagnóstico: IRA em PO de correção de cardio- } \\
\text { patia congênita }\end{array}$ & $\begin{array}{l}\text { Aumento da pressão da artéria pulmonar e atrial direi- } \\
\text { ta, sem alteração do índice cardíaco, RVS e pulmonar, } \\
\text { pressão atrial esquerda e saturação arterial de } \mathrm{O}_{2} \text {. }\end{array}$ \\
\hline
\end{tabular}
venosa central; RVS= resistência vascular sistêmica; SDRA= síndrome do desconforto respiratório agudo. 


\section{DISCUSSÃO}

A maior parte dos estudos ${ }^{13-18}$ abordam diferentes modalidades de diálise e comparam a DP com a hemofiltração, ou a DP com a hemodiálise, ou a hemodiálise com a hemofiltração. Entretanto, poucos estudos (Tabelas 2 e 3) analisaram as repercussões cardiorrespiratórias em pacientes pediátricos graves durante e após a DP.

No que se refere às repercussões no sistema respiratório, durante a DP em Pediatria, o estudo de Bunchman e col. ${ }^{19}$ avaliaram quatro crianças com diagnóstico clínico de insuficiência renal aguda e evidenciou redução da complacência pulmonar dinâmica, $\mathrm{PaO}_{2}$, aumento da resistência das vias aéreas e da $\mathrm{PaCO}_{2}$ durante o procedimento. Estas repercussões durante a DP podem ser explicadas pelos seus efeitos fisiológicos, pois ocorre o aumento da pressão intra-abdominal, decorrente da infusão ou aprisionamento de liquido na cavidade abdominal. $\mathrm{O}$ aumento da pressão intraabdominal ocasiona um aumento no retorno venoso, quando a pressão transmural da veia cava inferior (em nível torácico) supera a pressão de fechamento crítico transmural ${ }^{20}$. Associado a isto pode ocorrer diminuição da ventilação e subseqüentes alterações dos gases sangüíneos devido a diminuição da pressão transdiafragmática, podendo ocasionar atelectasias pulmonares $^{21,22}$. Por outro lado, o estudo de Morris, Butt e Karl ${ }^{23}$ não evidenciaram nenhuma repercussão neste sistema durante a DP.

Dentre os três artigos ${ }^{24-26}$ que avaliaram as complicações respiratórias após a DP, o estudo de Bokariia e col. ${ }^{24}$ identificaram que as 19 crianças (idade de 6,2 \pm 4,3 meses; peso de 6,1 $\pm 1,7 \mathrm{~kg}$ ) avaliadas, com diagnóstico clínico de IRA por disfunção de múltiplos órgãos ou cardiopatias congênitas, apresentaram vantagens positivas após 4 a 5 dias de DP, dentre elas: melhora hemodinâmica, trocas gasosas e das funções respiratórias. Neste estudo não foram identificadas repercussões clínicas após a infusão de líquidos pela DP.

No estudo retrospectivo de Werner e col. ${ }^{25}$, foram avaliadas 32 crianças (idade de $22 \pm 35$ meses) com diagnostico de IRA após cirurgia cardíaca no período de cinco anos. Esta modalidade foi considerada efetiva no pós-operatório cardíaco, pois houve redução da pressão média de vias aéreas e da diferença alvéoloarterial de oxigênio após a DP. As repercussões cardiorrespiratórias foram decorrentes do acúmulo de fluidos associadas ao processo pós-operatório de cirurgia cardíaca e a utilização de fármacos não demonstrando relação com a DP. Sagy e Silver ${ }^{26}$ avaliaram seis crianças (idade de 18,7 \pm 37 meses) com anasarca grave e síndrome do desconforto respiratório agudo, em ventilação pulmonar mecânica, após a DP, observaram um aumento da relação $\mathrm{PaO}_{2} / \mathrm{FiO}_{2}$, redução da diferença alvéolo-arterial de oxigênio e do índice de oxigenação nestes pacientes. Não foram encontradas complicações respiratórias decorrentes da DP ou infecciosas relacionadas ao cateter.

Dentre os quatro artigos ${ }^{23,27-29}$ que avaliaram as repercussões cardiocirculatórias durante a DP todos relataram alterações hemodinâmicas nos paciente pediátricos, dentre elas: aumento da PAM, pressão arterial pulmonar, do átrio esquerdo, átrio direito e aumento da pressão venosa central. Estas repercussões são decorrentes da administração de dialisato, ocorrendo aumento pressórico intra-abdominal, podendo predispor a criança à repercussões cardiocirculatórias.

Com base em modelo teórico e experimental ${ }^{20}$, foi descrito o conceito de zonas distintas de vascularização abdominal (similar às zonas de West no pulmão ${ }^{12}$ ). $\mathrm{O}$ aumento da pressão intra-abdominal reduz o retorno venoso quando a pressão transmural da veia cava inferior permanece abaixo da pressão de fechamento crítico transmural.

O estudo de Morris, But e Karl ${ }^{23}$ compararam os efeitos hemodinâmicos antes e durante a infusão de diferentes volumes na cavidade abdominal em seis crianças no pós-operatório cardíaco. Identificaram que após a administração de diversos volumes de dialisato $(0,10$, 20 e $30 \mathrm{~mL} / \mathrm{kg}$ ) o índice cardíaco foi maior com 20 e 30 $\mathrm{mL} / \mathrm{kg}$ quando comparado com a cavidade abdominal vazia ou com $10 \mathrm{~mL} / \mathrm{kg}$. Durante a administração dos diversos volumes de dialisato houve aumento da pressão intra-abdominal, entretanto, este aumento não esteve relacionado com repercussões cardiocirculatórias.

Dentre os estudos ${ }^{24,25,30,31}$ que avaliaram os efeitos cardiocirculatórios após a DP, o estudo de Werner e col..25 relataram aumento da PAM e da pressão venosa central em crianças em pós-operatório de cirurgia cardíaca. Os estudos de Bokariia e col. ${ }^{24}$ e Zhovnir e col. ${ }^{30}$ não identificaram repercussões do procedimento, verificando que após a DP houve melhora nos parâmetros hemodinâmicos e renais.

\section{CONCLUSÃO}

As repercussões cardiorrespiratórias mais freqüentes encontradas neste estudo foram as alterações dos volumes pulmonares e dos gases sanguíneos durante a 
DP, as alterações da pressão arterial média, da pressão venosa central, da resistência vascular sistêmica durante e após a DP. Estas repercussões devem ser monitoradas para se evitar a piora clínica e para a orientação das intervenções em crianças graves.

\section{REFERÊNCIAS}

01. Ajzen H, Schor N - Guia de Medicina Ambulatorial e Hospitalar UNIFESP - Escola Paulista de Medicina: Nefrologia. 2a Ed, São Paulo: Manole, 2005.

02. Schor N, Boim MA, Santos OFP - Insuficiência Renal Aguda: Fisiopatologia, Clínica e Tratamento. São Paulo; Sarvier, 1997.

03. Yu L, Abensur H - Diretrizes da Insuficiência Renal Aguda. Sociedade Brasileira de Nefrologia 2001. Disponível em: URL: http://www.sbn.org. br/Diretrizes/ira.htm.

04. Miller NL, Finberg L - Peritoneal dialysis for salt poisoning: report of a case. N Engl J Med, 1960;29:1347-1350.

05. Carvalho WB, Hirschheimer MR, Matsumoto T - Terapia Intensiva Pediátrica. $3^{a}$ Ed, São Paulo; Atheneu, 2006.

06. Bakkaloglu SA, Ekim M, Sever L et al - Chronic peritoneal dialysis in Turkish children: a multicenter study. Pediatr Nephrol, 2005;20:644-651.

07. Carvalho WB, Souza N, Souza RL - Emergência em Terapia Intensiva Pediátrica. $2^{\mathrm{a}}$ Ed, São Paulo: Atheneu, 2001.

08. Carvalho ES, Carvalho WB - Terapêutica e Prática Pediátrica, $2^{\mathrm{a}}$ Ed, São Paulo: Atheneu, 2000.

09. Duranti R, Laffi G, Misuri G et al - Respiratory mechanics in patients with tense cirrhotic ascites. Eur Respir J, 1997;10:1622-1630.

10. Fischbach M, Terzic J, Laugel V et al - Measurement of hydrostatic intraperitoneal pressure: a useful tool for the improvement of dialysis dose prescription. Pediatr Nephrol, 2003;18:976-980.

11. Cullen DJ, Coyle JP, Teplick R et al - Cardiovascular, pulmonary, and renal effects of massively increased intra-abdominal pressure in critically ill patients. Crit Care Med, 1989;17:118-121.

12. Cook DJ, Guyatt GH, Laupacis A et al - Rules of evidence and clinical recommendations on the use of antithrombotic agents. Chest, 1992;102:(Suppl4):305S-311S.

13. Bunchman TE.- Acute peritoneal dialysis access in infant renal failure. Perit Dial Int, 1996;16:(Suppl1):S509-S511.

14. Zobel G, Ring E, Muller W - Continuous arteriovenous hemofiltration in premature infants. Crit Care Med, 1989;17:534-536.

15. Moghal NE, Brocklebank JT, Meadrow SR - A review of acute renal failure in children: incidence, etiology, and outcome. Clin Nephrol,
1998;49:91-95.

16. Gallego N, Perez-Caballero C, Gallego A et al - Prognosis of patients with acute renal failure without cardiopathy. Arch Dis Child, 2000;84:258-260

17. Lowrie $L$ - Renal replacement therapies in pediatric multiorgan dysfunction syndrome. Pediatr Nephrol, 2000;14:6-12.

18. Fleming $\mathrm{F}$, Bohn $\mathrm{D}$, Edwards $\mathrm{H}$ et al - Renal replacement therapy after repais of congenital heart disease in children: a comparation of hemofiltration and peritoneal dialysis. J Thorac Cardiovasc Surg, 1995;109:322-331.

19. Bunchman TE, Meldrum MK, Meliones JE et al - Pulmonary function variation in ventilator dependent critically ill infants on peritoneal dialysis. Adv Perit Dial, 1992;8:75-78.

20. Takata M, Wise RA, Robotham JL - Effects of abdominal pressure on venous return: abdominal vascular zone conditions. J Appl Physiol, 1990;69:1961-1972.

21. West JB - Fisiologia Respiratória. São Paulo: Manole, 2000.

22. Leff AR, Schumacker PT - Fisiologia Respiratória: Fundamentos e Aplicações. Rio de Janeiro: Interlivros, 1996.

23. Morris KP, Butt WW, Karl TR - Effect of peritoneal dialysis on intra-abdominal pressure and cardio-respiratory function in infants following cardiac surgery. Cardiol Young, 2004;14:293-298.

24. Bokariia LA, larustovskiI MB, Grigor'iants RG et al - Peritoneal dialysis in the newborn and infants after radical correction of complex congenital heart defects. Anesteziol Reanimatol, 2002;2:42-48.

25. Werner HA, Wensley DF, Lirenman DS et al - Peritoneal dialysis in children after cardiopulmonary bypass. J Thorac Cardiovasc Surg, 1997;113:64-70.

26. Sagy M, Silver P - Continuous flow peritoneal dialysis as a method to treat severe anasarca in children with acute respiratory distress syndrome. Crit Care Med, 1999;27:2532-2536.

27. Dittrich S, Vogel M, Dahnert I et al - Acute hemodynamic effects of post cardiotomy peritoneal dialysis in neonates and infants. Intensive Care Med, 2000;26:101-104.

28. Bunchman TE, McBryde KD, Mottes TE et al - Pediatric acute renal failure: outcome by modality and disease. Pediatr Nephrol, 2001;16:1067-1071.

29. Ryan CA, Hung O, Soder CM - Hemodynamic affects of peritoneal dialysis in three children following open heart surgery. Pediatr Cardiol, 1992; 13: 30-32.

30. Zhovnir VA, Emets IN, Mazur AR et al - The experience of application of peritoneal dialysis in cardiosurgery of an early age children. Klin Khir, 2000;8:31-33.

31. Dittrich S, Dähnert I, Vogel $M$ et al - Peritoneal dialysis after infant open heart surgery: observations in 27 patients. Ann Thorac Surg, 1999;68:160-163. 